\title{
Sustained-release of FGF-2 from a hybrid hydrogel of heparin-poloxamer and decellular matrix promotes the neuroprotective effects of proteins after spinal injury
}

This article was published in the following Dove Press journal:

International Journal of Nanomedicine

\author{
He-Lin Xu ${ }^{1, *}$ \\ Fu-Rong Tian ${ }^{1, *}$ \\ Jian Xiaol,* \\ Pian-Pian Chen' \\ Jie $X u^{\prime}$ \\ Zi-Liang Fan' \\ Jing-Jing Yang' \\ Cui-Tao Lu' \\ Ying-Zheng Zhao ${ }^{1,2}$ \\ 'Department of Pharmaceutics, School \\ of Pharmaceutical Sciences, Wenzhou \\ Medical University, Wenzhou, ${ }^{2}$ Hainan \\ Medical College, Haikou, China \\ *These authors contributed equally \\ to this work
}

\begin{abstract}
Introduction: The short lifetime of protein-based therapies has largely limited their therapeutic efficacy in injured nervous post-spinal cord injury (post-SCI).

Methods: In this study, an affinity-based hydrogel delivery system provided sustained-release of proteins, thereby extending the efficacy of such therapies. The affinity-based hydrogel was constructed using a novel polymer, heparin-poloxamer (HP), as a temperature-sensitive bulk matrix and decellular spinal cord extracellular matrix (dscECM) as an affinity depot of drug. By tuning the concentration of HP in formulation, the cold ternary fibroblast growth factor-2 (FGF2)-dscECM-HP solution could rapidly gelatinize into a hydrogel at body temperature. Due to the strong affinity for FGF2, hybrid FGF2-dscECM-HP hydrogel enabled sustained-release of encapsulated FGF2 over an extended period in vitro.
\end{abstract}

Results: Compared to free FGF2, it was observed that both neuron functions and tissue morphology after SCI were clearly recovered in rats treated with FGF2-dscECM-HP hydrogel. Moreover, the expression of neurofilament protein and the density of axons were increased after treatment with hybrid FGF2-dscECM-HP. In addition, the neuroprotective effects of FGF2-dscECM-HP were related to inhibition of chronic endoplasmic reticulum stress-induced apoptosis.

Conclusion: The results revealed that a hybrid hydrogel system may be a potential carrier to deliver macromolecular proteins to the injured site and enhance the therapeutic effects of proteins.

Keywords: spinal cord injury, decellularized extracellular matrix, thermosensitive hydrogel, adsorption, basic fibroblast growth factor

\section{Introduction}

Spinal cord injury (SCI), one of the most devastating traumas, leads to sudden loss of sensory, motor, and autonomic functions distal to the level of injury. ${ }^{1}$ Experimental studies have shown that pathological processes after SCI consist of primary and secondary damages. Primary mechanical injury to the spinal cord triggers a cascade of molecular events, which tend to cause severe secondary damage, such as disturbances in ionic homeostasis, local edema, ischemia, focal hemorrhage, free radical stress, inflammatory response, necrosis, and apoptosis. ${ }^{2-4}$ Current treatment includes decompression surgery and post-injury stabilization of physiological conditions, prevention and management of secondary complications, and rehabilitation. However, neurological recovery is limited, and substantial neurological dysfunction and lifelong disability still afflict most SCI patients.
Correspondence: Cui-Tao Lu; Ying-Zheng Zhao

Department of Pharmaceutics, School of Pharmaceutical Sciences, Wenzhou Medical University, Wenzhou,

Zhejiang, 325035, China

Tel +865778669 9575

Email lctuua@sina.com; pharmtds@163.com

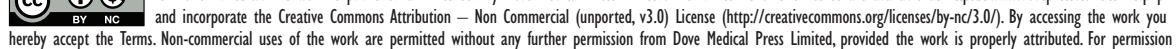
for commercial use of this work, please see paragraphs 4.2 and 5 of our Terms (https://www.dovepress.com/terms.php). 
After SCI, harmful cascades, such as neuronal death, activation of astrogliosis, and inflammation, are the most important factors preventing repair of injured neurons. Lesioned spinal cords usually induced the formation of marked hypertrophy and proliferation of astrocytes, known as excessive activation of astrocytes, which activated the expression of glial fibrillary acidic protein (GFAP) and axon growth inhibitory chondroitin sulfate proteoglycans (CSPGs). These phenotypic changes in astrocytes are considered to be harmful factors for neurological recovery after SCI. Basic fibroblast growth factor (bFGF or FGF2), a member of the FGF family, has multiple roles in functional recovery after SCI. A previous report that evaluated the effect of FGF2 in a hemisection rat SCI model indicated that the effect of FGF2 on SCI is related to inhibited inflammation, lower production of CSPG, and increased glial processes. ${ }^{5}$ However, the effect of FGF2 on SCI was associated with the proliferation, morphogenesis, and apoptosis of various cell populations, particularly astrocytes and neural cells, via complex signal transduction pathways in these studies. ${ }^{6-8}$ The beneficial effects of FGF2 on SCI were correlated with neuronal regeneration and functional recovery. FGF2 not only exhibited the neuroprotective and neurotrophic effect but was also involved in the inhibition of excessive astrogliosis and glial scarring after neuronal injury. For example, our previous studies showed that exogenous FGF2 attenuated astrocyte activation by reducing the expression of GFAP and other markers, including neurocan and vimentin, via the regulation of the upstream toll-like receptor 4/nuclear factor $\mathrm{KB}$ (TLR4/NFKB) signaling pathway. ${ }^{9}$ More importantly, FGF2 had many positive effects on neural cell populations. FGF2 increased the survival of mature neurons in vitro, promoted the survival and proliferation of neural stem cells, and maintained them in an undifferentiated state. For example, the transient infusion of FGF2 could stimulate the growth of axon regeneration and inhibit the apoptosis of neurons in secondary damage after SCI. However, since FGF2 as a macromolecular protein had poor penetrability of the blood-spinal cord barrier and a short half-life in the body, the therapeutic efficacy of FGF2 on injured nervous tissue was limited via either subcutaneous or intravenous administration. Multiple injections or implantation of semi-permanent cannulas may bypass this limitation but require invasive surgical techniques that limit their clinical relevance. ${ }^{10,11}$ Therefore, it is urgently required to identify a more effective route of FGF2 administration to maintain an effective concentration of FGF2 in injured spinal cord.
In situ delivery of FGF2 using biomaterials may not only support injured tissue but also maintain a sustained-release of encapsulated drugs. Three-dimensional hydrogels had good affinity and compatibility with biological tissue due to their high water content. With the proper formula, hydrogels can load biological macromolecules to achieve controlled release in situ. A variety of hydrogels (natural or synthetic) have been proposed as scaffolds that can allocate growth factors. A drawback for most natural or synthetic hydrogels that do not appropriately represent a biological niche is a lack of affinity with growth factors allocated in the hydrogel matrix, which results in rapid diffusion and activity loss of encapsulated FGF2. Recently, affinity-based biomaterial delivery systems have been observed to provide sustained-release of growth factors, thereby improving their therapeutic efficacy. ${ }^{12-14} \mathrm{~A}$ novel heparin-poloxamer (HP) hydrogel, which had an affinity for growth factors and a tuneable-phase transition temperature for use in the injured spinal cord, was prepared in our previous study. ${ }^{15,16}$ However, HP hydrogel lacked a micro-niche to store and stabilize growth factors as they were secreted in the body, making them incapable of attracting or attaching growing cells to injured sites in vivo, which is required to promote the regeneration of injured tissues. Decellular spinal cord extracellular matrix ( $\mathrm{dscECM}$ ) has been demonstrated to be a crucial element to promote the adhesion, trophic support, and survival of growing cells. Due to its good biocompatibility with many neural tissues and its soft and flexible texture, dscECM can be used as a potential biomaterial in the field of SCI repair and regeneration. Recently, many studies have used dscECM as a cell carrier to promote neural recovery after SCI. ${ }^{17}$ In addition, FGF2 antibodies, which can sequester exogenous FGF2, were also observed in dscECM, making them an ideal micro-depot for FGF2. ${ }^{18}$

In this study, a hybrid hydrogel was constructed by incorporating dscECM into the HP solution. By tuning the concentration of HP, the mixed dscECM-HP solution could rapidly transit from a liquid status to a semi-solid hydrogel at body temperature. The ability of dscECM to store and sequester FGF2 was evaluated by in vitro affinity experiments. The FGF2-dscECM depot was added to the cold HP solution, and the in vitro characteristics of the FGF2-dscECM-HP ternary system were carefully evaluated. The attachment and growth of PC12 cells in the FGF2-dscECM-HP hybrid hydrogel were also evaluated in vitro. Finally, the therapeutic effect of the FGF2-dscECM-HP hybrid hydrogel on SCI rats was explored, and the relationship between the anti-apoptotic effect of FGF-2 and functional recovery in SCI model was investigated. 


\section{Materials and methods Materials}

HP was synthesized according to the method depicted in our previous study. ${ }^{19}$ Basic fibroblast growth factor (FGF2) was ordered from Gelusite Biology Technology Company (Zhe jiang, China). Fluoresceins were ordered from SigmaAldrich Co. (St Louis, MO, USA). All antibodies, including anti-NF200, anti-Neuron, and caspase-3, were purchased from Abcam (Cambridge, UK). Biotinylated dextran amine (BDA) was purchased from Thermo Fisher Scientific (Waltham, MA, USA). Healthy female Sprague-Dawley rats (220-250 g) were purchased from the SLAC Laboratory Animal Corporation (Shanghai, China).

\section{Preparation and characterization of dscECM}

FGF2-dscECM-HP hydrogels were prepared using the cold method. ${ }^{20}$ Briefly, the spinal cord was minced, rinsed in $0.01 \%$ phosphate-buffered saline (PBS, $\mathrm{pH} 7.4$ ) for $1 \mathrm{~h}$, and treated with $1 \%$ of Triton X-100 solution for $12 \mathrm{~h}$. The decellularized spinal cord tissue was washed three times using sterile distilled water (10 min each, $60 \mathrm{rpm}$ ) followed by treatment with $4.0 \%$ deoxycholate for $8 \mathrm{~h}$. Finally, the dscECM was washed using PBS ( $\mathrm{pH}$ 7.4) for at least 3 days to remove residual detergent, sterilized using $0.1 \%$ peracetic acid (Sigma-Aldrich Co.) in 4\% ethanol for $4 \mathrm{~h}$, and washed several times with filtered PBS. The dscECM was carefully characterized by $\mathrm{HE}$ and immunofluorescence staining, and after homogenization, its components were quantitatively detected by the corresponding enzyme-linked immunosorbent assay (the methods are found in the Supplementary materials).

\section{Preparation and characterization of FGF2-dscECM-HP hydrogel}

The dscECM was further homogenized in $0.5 \mathrm{M}$ acetic acid for $1 \mathrm{~h}$ and was digested after addition of pepsin powder (Sigma-Aldrich Co.) at room temperature for $24 \mathrm{~h}$. After solubilization, PBS (10 mM) was added to adjust ionic balance. To remove undigested particles, the dscECM solution was filtered through a $40-\mu \mathrm{m}$-pore mesh. The dscECM solution in PBS was further lyophilized, and the lyophilized powder was stored at $-20^{\circ} \mathrm{C}$ until use. The lyophilized dscECM powder was added to the FGF2 solution and completely dissolved after incubation for $24 \mathrm{~h}$ at $4^{\circ} \mathrm{C}$ under gentle stirring. Finally, HP powder ( $0.8 \mathrm{~g}$ ) was dissolved in $5 \mathrm{~mL}$ of FGF2-dscECM solution and mixed at $4^{\circ} \mathrm{C}$ with gentle stirring overnight. The mixture was kept in a refrigerator at $4^{\circ} \mathrm{C}$ overnight until a clear solution was formed. The final concentrations of FGF2 and dscECM in FGF2-ECM-HP hydrogel were adjusted to 3 and $10 \mathrm{mg} / \mathrm{mL}$, respectively.

\section{Screening of HP concentration in dscECM-HP}

To screen the optimal concentration of HP, FGF2dscECM-HP solutions with different HP concentrations were placed in a thermostatic water bath and heated at a rate of $0.5^{\circ} \mathrm{C} / \mathrm{min}$ (from $22^{\circ} \mathrm{C}$ up to $60^{\circ} \mathrm{C}$ ). The solution-gel transition temperature of FGF2-dscECM-HP solution at which the immobility of the meniscus in each vial was first noted was recorded. ${ }^{21}$ Meanwhile, the apparent viscosity of the FGF2dscECM-HP solution was also tested in a coaxial cylinder rheometer (DV-III; Brookfield, Middleborough, MA, USA) with an SC4-14 rotor and a small sample adapter. Curves of apparent viscosity at different temperatures were obtained.

\section{Scanning electron microscopy (SEM)}

The morphology of the FGF2-dscECM-HP hydrogel was determined using SEM (X-650; Hitachi Ltd., Tokyo, Japan). The FGF2-dscECM-HP hydrogel was lyophilized using standard processing and directly observed by SEM. To observe the morphology of dscECM, the intact dscECM scaffold was lyophilized, cross-sectioned, and sputter-coated with gold for SEM observation.

\section{Affinity of dscECM-HP to FGF2}

The homogenized dscECM (100 mg dry weight) was first mixed with $1 \mathrm{~mL}$ of cold $16 \% \mathrm{HP}$ solution in an ice bath to prepare a mixed dscECM-HP solution. Afterward, incubation at $37^{\circ} \mathrm{C}$ gelatinized the dscECM-HP solution. To study the affinity of FGF2 to hybrid dscECM-HP hydrogel, $1 \mathrm{~mL}$ of FGF2 solution ( $1 \mathrm{mg} / \mathrm{mL})$ was added to the surface of the hybrid dscECM-HP hydrogel and further incubated for $24 \mathrm{~h}$ at $37^{\circ} \mathrm{C}$. The supernatant was withdrawn, and hybrid dscECM-HP hydrogel was rinsed three times with $1 \mathrm{~mL}$ of warm PBS. The total amount of FGF2 in all supernatants was quantified using its absorbance at $280 \mathrm{~nm}$. As a control, $16 \%$ of HP hydrogel alone was used as a control, and its absorption was also analyzed according to the methods described above.

\section{FGF2 release from hybrid FGF2-dscECM- HP hydrogel}

To trace the FGF2 distribution in various in vitro experiments, FGF2 was covalently labeled with fluorescein isothiocyanate (FITC) using the method described in the literature. ${ }^{18,22}$ FITC-labeled FGF2 (FITC-FGF2) replaced FGF2 to prepare 
the fluorescent hybrid hydrogel (FITC-FGF2-dscECM-HP) for the in vitro study of its release. To detect FGF2 release from temperature-sensitive FGF2-dscECM-HP, FITClabeled FGF2 was encapsulated into dscECM-HP to prepare a fluorescent FITC-FGF2-dscECM-HP hydrogel. The in vitro release method was described previously in the literature ${ }^{23,24}$ with several modifications. Briefly, $1 \mathrm{~mL}$ of cold FITC-FGF2-dscECM-HP solution was placed in tubes and allowed to gel completely at $37^{\circ} \mathrm{C}$. Next, $1 \mathrm{~mL}$ of $\mathrm{pH}$ 7.4 PBS was added to the hydrogel samples, and the tubes containing hydrogel were placed in a vibrating incubator at $37^{\circ} \mathrm{C}$ under continuous shaking at a speed of $80 \mathrm{rpm} / \mathrm{min}$. At predetermined time intervals, $300 \mu \mathrm{L}$ of the released medium was sampled and replaced by an equal volume of fresh medium to maintain a constant volume. The detected fluorescence intensity of the prepared FITC-FGF2 $\left(\mathrm{I}_{\text {total }}\right)$ contained the same volume of released medium as the total drug amount, and the fluorescence intensity of samples at different time points $\left(\mathrm{I}_{t}\right)$ was quantified using a Thermo Scientific Microplate Reader at $\lambda_{\mathrm{ex}}=495 \mathrm{~nm}$ and $\lambda_{\mathrm{em}}=525 \mathrm{~nm}$. The cumulative release rate of FITC-FGF2 from the FITCFGF2-dscECM-HP hydrogel in vitro was calculated according to the following formula:

$$
\text { Cumulative release rate }(\%)=\frac{\sum \mathrm{I}_{\mathrm{t}}}{\mathrm{I}_{\text {total }}} \times 100 \%
$$

\section{Cell attachment and growth in an FGF2-dscECM-HP hydrogel}

\section{Cell culture}

PC12 cells were purchased from the Cell Storage Center of Wuhan University (Wuhan, China) and approved by the Ethics Committee of Wenzhou Medical University in this study. PC12 cells were cultured in high-glucose Dulbecco's Modified Eagle's Medium with 10\% fetal bovine serum and $1 \%$ penicillin-streptomycin in a humidified incubator containing $5 \% \mathrm{CO}_{2}$ at $37^{\circ} \mathrm{C}$. The cells in their logarithmic growth phase were harvested with trypsin for further experiments.

\section{Cell proliferation and apoptosis in FGF2-dscECM-HP hydrogel}

The cytotoxicity and proliferation of dscECM-HP against PC12 cells were measured using a cell counting kit-8 (CCK-8, MedChemExpress, Monmouth Junction, New Jersey, USA). PC12 cells were cultured in a 96-well plate at a density of 5,000 cells per well for $24 \mathrm{~h}$. The medium was replaced with thapsigargin (TG) solution to induce cell injury. Although there is no TG in the body, endoplasmic reticulum stress induced by TG in PC12 cells can result in cell apoptosis. After $24 \mathrm{~h}$ of incubation, the supernatant culture medium was removed and replaced with fresh medium (control), dscECM-HP hydrogel, bFGF solution, and FGF2-dscECM-HP hydrogel for different treatment groups, respectively. The FGF2 concentration in all treatment groups was $40 \mathrm{ng} / \mathrm{mL}$. After $24 \mathrm{~h}$ of treatment, the supernatant medium was discarded, and $10 \mu \mathrm{L}$ of CCK- 8 solutions were added to the wells and incubated for $2 \mathrm{~h}$ to quantify cell proliferation. The absorbance was measured at $450 \mathrm{~nm}$ with a reference wavelength of $650 \mathrm{~nm}$. Meanwhile, the apoptotic rates of the PC12 cells were also measured using a PI/Annexin V-FITC kit (Thermo Fisher Scientific) and analyzed with a FACS scan flow cytometer (Becton Dickinson, Franklin Lakes, NJ, USA) for the manual description.

\section{Cell attachment to FGF2-dscECM-HP hydrogel}

To observe cell attachment in the hydrogel, PC12 cells $\left(1 \times 10^{6}\right.$ cells) were first labeled with fluorescein (Synonym, CM-DiI) and added to the surface of an FGF2-dscECM-HP hydrogel $(1 \mathrm{~mL})$ layer on a glass-bottom dish. After $6 \mathrm{~h}$ of culture, the free cells were withdrawn, and the hydrogel was washed with warm PBS three times. The attached cells were observed under a confocal laser scanning microscope. The HP hydrogel was used as a control and was evaluated by the procedure described above.

\section{FGF2-dscECM-HP hydrogel promotes functional recovery of $\mathrm{SCl}$ rats $\mathrm{SCl}$ rat model and drug treatments}

This study was performed in strict accordance with the recommendations in the Guide for the Care and Use of Laboratory Animals of the National Institutes of Health. All animal experiments were performed with the approval and according to the guidance of the Institutional Animal Care and Use Committee of Wenzhou Medical University (Permit Number: XI104079). Healthy female SpragueDawley rats (220-250 g) were purchased from the SLAC Laboratory Animal Corporation and housed at $23^{\circ} \mathrm{C} \pm 2{ }^{\circ} \mathrm{C}$ and humidity of $50 \% \pm 10 \%$ controlled with a $12 \mathrm{~h}$ light $/ 12 \mathrm{~h}$ dark cycle. Animals were anesthetized by an intraperitoneal injection of pentobarbital sodium $(60 \mathrm{mg} / \mathrm{kg})$ and placed on a constant temperature heating platform. After shaving the hair and removing muscle at the thoracic level, a laminectomy was performed on thoracic vertebrae 9-10 (T9-T10). An incision was made with a fine scalpel blade through the meningeal membranes, and a hemisection was introduced at the right side of the spinal cord, sparing only the left section. 
Sham group animals received the same surgical procedures, but resection was not carried out. All animals were randomly divided into different groups (12 rats per group). FGF2 solution/FGF2-HP hydrogel/FGF2-dscECM-HP hydrogel were injected at a dose of $20 \mu \mathrm{L}$ (FGF2 concentration is $3 \mu \mathrm{g} / \mu \mathrm{L}$ ) through a microsyringe after SCI. The rats of the sham group and SCI group were given the same dose of normal saline solutions. After surgery, animals were returned and received manual bladder expression twice daily until bladder function was restored.

In vivo FGF2 release from FGF2-dscECM-HP hydrogel In vivo spinal cord fluorescence imaging was detected by the Maestro in vivo optical imaging system (CRI, Inc., Woburn, MA, USA). ${ }^{25}$ To produce a fluorescence signal, FITC-labeled FGF2 (FITC-FGF2) was used in two types of hydrogels, FITC-FGF2-HP and FITC-FGF2-dscECM-HP. In our previous publication, FITC was covalently conjugated to FGF2 through amine bonds without compromising the physiochemical properties of FGF2, and there was a good correlation between the fluorescence signal intensity of FITC and FGF2 concentration. ${ }^{22}$ Thus, detection of FITC fluorescence was usually exploited to evaluate in vitro release of FGF2 from hydrogels and trace its distribution in vivo. ${ }^{26}$ Approximately $20 \mu \mathrm{L}$ of cold FITC-FGF2-HP solution and FITC-FGF2-dscECM-HP solution were injected into the injured spinal cord in situ. The rats were sacrificed, and their spinal cords were separated at $15 \mathrm{~min}$, $1,3,5$, and 7 days after the injection of the hydrogel. The residual FGF2 in the hydrogel was determined by observing the fluorescence intensity. Each group consists of three animals.

\section{Functional analysis}

The Basso, Beattie, and Bresnahan (BBB) scoring method was used to assess open field locomotor function ${ }^{27}$ at $0,3,7$, 14 , and 28 days. Two trained investigators who were blind to the experimental conditions scored the locomotion recovery in an open field according to the BBB scale. BBB scores ranged from 0 , indicative of no hind limb movements, to a score of 21, representative of a normal ambulating rodent. Animals were placed individually on open fields and allowed to move freely for $5 \mathrm{~min}$. The animals were evaluated every 2 days from day 0 to day 28 after the surgical procedures. The inclined plane test was used to test whether animals could continue to maintain a stable posture and measure the strength of the hind legs. The test was performed at $0,3,7$, 14, and 28 days. For each position, we recorded the maximum angle of the inclined plane at which the rats maintained a constant position for $5 \mathrm{~s}$.

\section{Neuroprotective effect of the FGF2-dscECM-HP hydrogel}

Histology, immunofluorescence, and immunohistochemical staining

The rats were deeply anesthetized with $1 \%$ pentobarbital sodium $(60 \mathrm{mg} / \mathrm{kg}$, intraperitoneal) and perfused with $0.9 \%$ $\mathrm{NaCl}$, followed by $4 \%$ paraformaldehyde in $0.01 \mathrm{M}$ PBS $(\mathrm{pH}=7.4)$ at $28 \mathrm{~d}$. The spinal cords were excised at the T8-T10 level, post-fixed in cold 4\% paraformaldehyde overnight, and embedded in paraffin. The spinal cord tissues were mounted in poly-L-lysine-coated slides for histopathological examination. Longitudinal paraffin sections $(5-\mu \mathrm{m}$ thick) were located in the central part of SCI. The sections were differentiated in 95\% ethyl alcohol, dehydrated in 100\% alcohol, and rinsed in xylene followed by staining in Nissl solution (Beyotime Biotechnology, Shanghai, China).

Immunohistochemistry staining was applied to detect protein expression. The $5-\mu \mathrm{m}$-thick paraffin sections were incubated in $3 \% \mathrm{H}_{2} \mathrm{O}_{2}$ for $15 \mathrm{~min}, 80 \%$ carbinol for $30 \mathrm{~min}$, and blocking solution for $1 \mathrm{~h}$ at room temperature. Subsequently, the sections were incubated at $4^{\circ} \mathrm{C}$ overnight with the following primary antibodies: polyclonal chicken anti-NF200 (Abcam; 1:10,000), polyclonal rabbit anti-CHOP (Abcam; 1:200), anti-Neuron (Abcam; 1:500) and anti-Caspase-3 (Abcam; 1:400). The paraffin sections were incubated with horseradish peroxidase-conjugated secondary antibodies for $2 \mathrm{~h}$ at $37^{\circ} \mathrm{C}$. The reaction was stopped with 3,3-diaminobenzidine. The paraffin sections were treated with FITC-conjugated secondary antibodies for $1 \mathrm{~h}$ at $37^{\circ} \mathrm{C}$, and the results were imaged by optical microscopy (Nikon ECLIPSE Ti-S, Ruikezhongyi Company, Beijing, China). The total number of positive staining cells on each representative T9 spinal cord section was counted for the SCI region using Image-Pro Plus software (Media Cybernetics, Inc., Rockville, MD, USA).

\section{Anterograde tracer}

Two weeks post-SCI, rats were anesthetized with chloral hydrate, and the procedures were performed as described in these publications..$^{28,29}$ In brief, after placing the animal in a stereotaxic apparatus, the skin was incised at the midline to expose the skull. Approximately $500 \mathrm{~nL}$ of a mixture of dextran amine conjugated with Texas Red (10\% BDA; MW 10,000; Thermo Fisher Scientific) was injected through a glass micropipette (diameter $\sim 50 \mathrm{~mm}$ ) at eight positions on the left hemisphere, approximately spanning the rostro-caudal 
extent of the hind limb sensorimotor cortex. BDA delivery took $5 \mathrm{~min}$ at each site. The micropipette tip remained in place for $20 \mathrm{~s}$ before withdrawal. After 2 weeks, the tissues were handled with color rendering and statistical processing.

\section{Western blot analysis}

Total protein was collected at the central tissue of SCI. Proteins were extracted in an ice bath, and their levels were assayed using standard biochemical procedures. ${ }^{30}$ Western blots were used to evaluate the polyclonal rabbit antimouse CHOP (Abcam; 1:1,000), the polyclonal rabbit antimouse GRP78 (Abcam; 1:1,000), the polyclonal rabbit anti-mouse Caspase-12 (Abcam; 1:1,000), and the polyclonal rabbit anti-mouse GAPDH (Abcam; 1:700) levels in experimental rats. Signals were visualized using a ChemiDoc ${ }^{\mathrm{TM}}$ XRS+ Imaging System (Bio-Rad), and the band densities were quantified by densitometry (Image Lab; Bio-Rad Laboratories Inc., Hercules, CA, USA).

\section{Statistical analysis}

The data were expressed as the mean \pm SD. One-way ANOVA and Student's $t$-test or Kruskal-Wallis test were adopted for statistical comparison using GraphPad Prism 5.0 (GraphPad Software, Inc., La Jolla, CA, USA). The difference was considered to be significant when the $P$-value was $\leq 0.05$.

\section{Results}

\section{Preparation and characterization of dscECM}

After decellularization of normal spinal cord, dscECM exhibited a somewhat transparent appearance. Normal spinal cord tissues had many integrated nuclei in the spinal cord cells (Figure S1A-C), whereas no residual cellular nuclei were visible in H\&E and DAPI images of the dscECM scaffold (Figure S1D-F). In addition, a pink-stained matrix with many cavities could be observed in the dscECM scaffold, indicating the deprivation of cells. With the same result from electron microscopy analysis, cells were not found in the scECM, but the integrity of the extracellular matrix was not disrupted and remained a three-dimensional network structure (Figure S1F). The residual DNA in the dscECM scaffold was further detected to confirm complete decellularization. As shown in Figure S1G, no residual DNA band appeared in the dscECM sample. Inversely, many continuous DNA bands were observed in normal spinal cord tissues. Several components of the dscECM, including protein-type and glycosaminoglycan (GAG)-type components, were also evaluated by immunofluorescence staining. According to $\underline{\text { Figure } \mathrm{S} 2}$, obvious positive reactions to collagen IV, laminin, fibronectin, and elastin were observed in dscECM, indicating the preservation of major protein-type components in dscECM. In addition, GAG was preserved following decellularization according to the results of periodic Acid-Schiff staining (Figure S2B). To investigate whether these components are lost during decellularization, these components were further quantified by enzyme-linked-immunosorbent serologic assay (ELISA). As shown in Figure S2C, the levels of collagen IV, laminin, and fibronectin in dscECM were comparable to those of the normal spinal cord reported in previous publications. ${ }^{31,32}$ The preservation of protein-based components may provide trophic support to promote the regeneration of injured nerve cells after SCI. More importantly, the dscECM scaffold retained a high level of GAGs, reaching 2.09 $\pm 0.12 \mu \mathrm{g}$ GAGs/mg dry weight, which benefitted the binding of growth factors for sustained-release.

\section{Preparation and characterization of FGF2-dscECM-HP hydrogel}

A novel copolymer-HP had good affinity to FGF2 and a controlled phase transition according to temperature variation. At lower temperatures, HP was in liquid phase, and it became a semisolid hydrogel when the temperature increased to a critical value (Figure 1A). The solution-gel transition was highly dependent on polymer concentration. To prepare a hydrogel with suitable properties for in situ application, the concentration of HP was first screened by measuring the apparent viscosity and solution-gel transition temperature of the HP solution. The apparent viscosity of the hydrogel first increased and then reached a plateau value with the change in temperature. The apparent viscosity at the plateau ranged from 7,000 to $16,000 \mathrm{mPa} \cdot \mathrm{s}$, depending on HP concentration. According to the literature, ${ }^{19}$ the apparent viscosity of the hydrogel suitable for in situ application was $\sim 10,000 \mathrm{mPa} \cdot \mathrm{s}$. Therefore, a 16\% (w/w) HP solution was the most suitable concentration; its apparent viscosity increased from 8,000 to $10,000 \mathrm{mPa} \cdot \mathrm{s}$ from $35^{\circ} \mathrm{C}$ to $37^{\circ} \mathrm{C}$ and maintained a stable viscosity of $10,000 \mathrm{mPa} \cdot \mathrm{s}$ at the plateau (Figure 1B). In addition, the process of gelation is instantaneous at $35^{\circ} \mathrm{C}$ to $\sim 37^{\circ} \mathrm{C}$, which will prevent the flow of liquid. By determining the gelation temperature of the HP solution with different concentrations, as HP concentration increased, the gelation temperature decreased and was tuneable, varying from $20^{\circ} \mathrm{C}$ to $60^{\circ} \mathrm{C}$ (Figure $1 \mathrm{C}$ ). Approximately $16 \%(\mathrm{w} / \mathrm{w}$ ) of the HP solution exhibited a rapid solution-to-hydrogel transition at $35^{\circ} \mathrm{C}-37^{\circ} \mathrm{C}$. This property is very important for the in situ hydrogel to be retained in injured tissue 
A

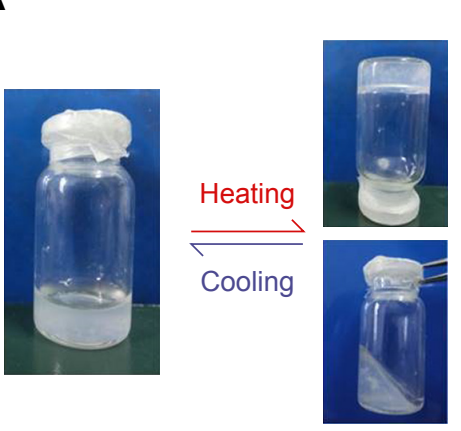

D

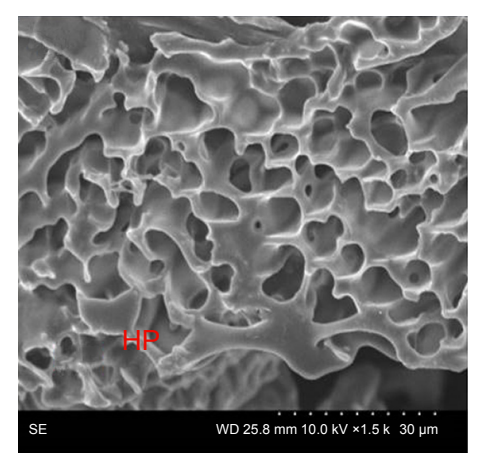

B
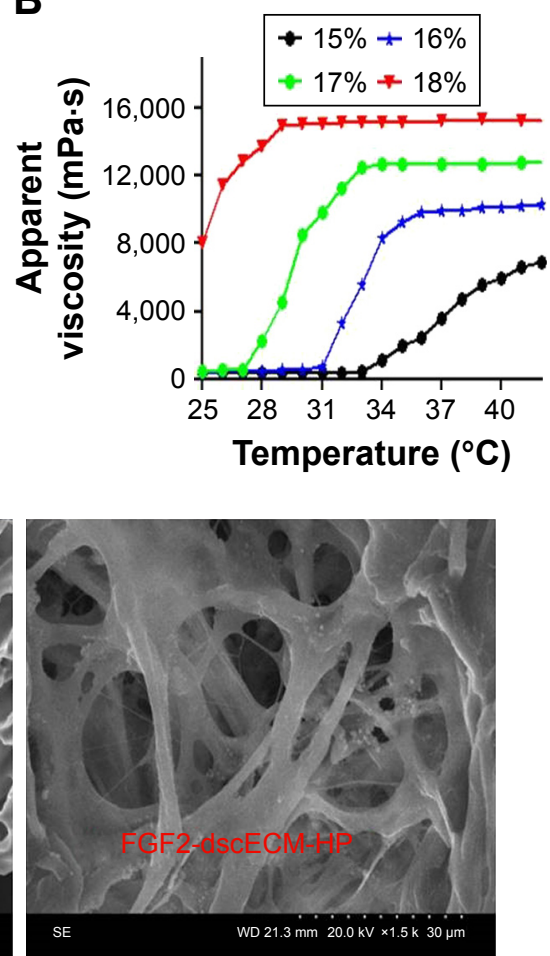

C

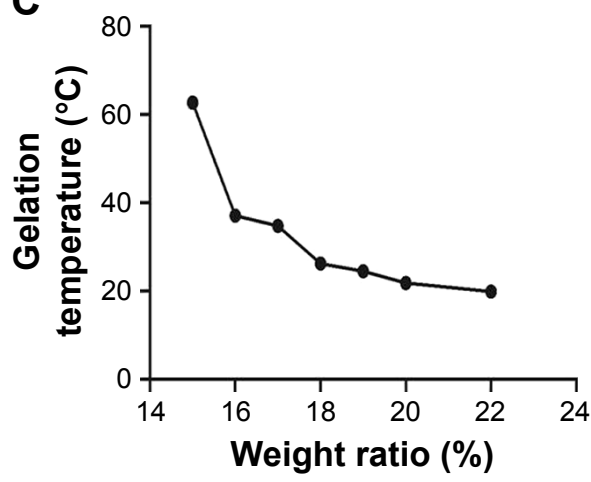

$E$

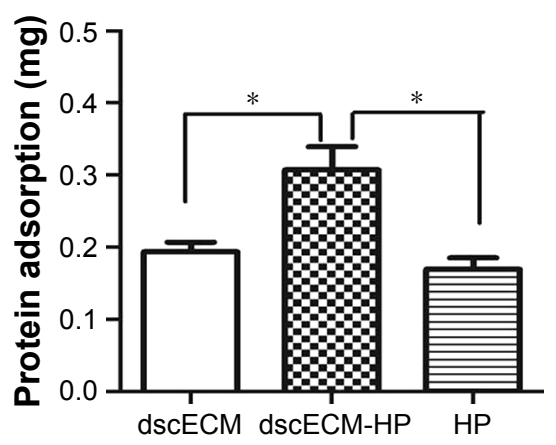

Figure I Characterization of FGF2-dscECM-HP hydrogel: (A) solution-gel transition of HP solution as temperature increased, (B) apparent viscosity of dscECM-HP as a function of HP concentration, (C) solution-gel transition temperature of HP solution with different concentrations, (D) SEM image of HP hydrogel and FGF2-dscECM-HP hydrogel, and (E) FGF2 adsorption by dscECM-HP after incubation for $24 \mathrm{~h}$ at $37^{\circ} \mathrm{C}$. $* P<0.05$.

Abbreviations: FGF2, fibroblast growth factor-2; dscECM, decellular spinal cord extracellular matrix; HP, heparin-poloxamer; SEM, scanning electron microscopy.

after administration. From SEM analysis, the HP hydrogel showed a porous network (Figure 1D), in which the inner pores of the hydrogel were interconnected. Compared to the HP hydrogel, the FGF2-dscECM-HP hydrogel still showed a porous structure, indicating that dscECM did not clearly affect the structure of the HP hydrogel. The porous network structure was also beneficial for the sustained-release of bFGF from the hydrogel; dscECM has been demonstrated to sequester growth factors and provide a micro-niche for their stabilization in a previous study. ${ }^{18}$ Therefore, the enhanced affinity of FGF2 was expected when HP hydrogel combined with dscECM. Indeed, the adsorption study showed that the dscECM-HP hydrogel exhibited twice as much adsorbed FGF2 as that of the HP hydrogel alone (Figure 1E), which indicated that dscECM also played an important role in affinity to FGF2. It was easily inferred that dscECM served as a micro-niche depot for FGF2 when it was preincubated with dscECM in advance before addition to the HP solution.

\section{FGF2 release from the FGF2-dscECM-HP hydrogel in vitro}

In vitro release curves of FGF2 from the FGF2-dscECM-HP hydrogel and FGF2-HP hydrogel are depicted in Figure 2.
The FGF2-HP hydrogel exhibited a rapid release profile for FGF2, with the cumulative release of FGF2 reaching $58 \%$ and $65 \%$ within 12 and $24 \mathrm{~h}$, respectively. In contrast, a slower release of FGF2 from the FGF2-dscECM-HP hydrogel was observed, with only $35 \%$ and $47 \%$ of embedded FGF2 being released within 12 and $24 \mathrm{~h}$, respectively. These results indicate that the affinity interaction between

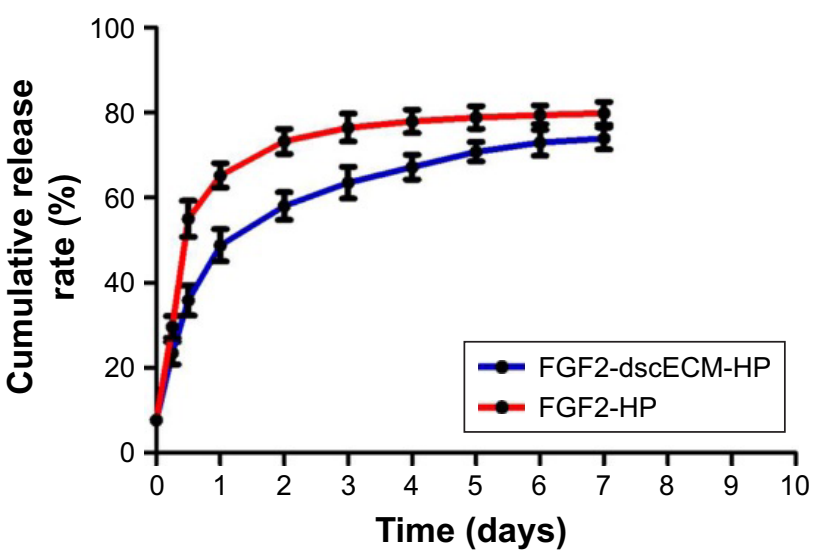

Figure 2 In vitro cumulative release of FGF2 from FGF2-dscECM-HP hydrogel and FGF2-HP hydrogel (data presented as mean $\pm S D, n=3$ ).

Abbreviations: FGF2, fibroblast growth factor-2; dscECM, decellular spinal cord extracellular matrix; HP, heparin-poloxamer. 
FGF2 and dscECM could prohibit the release of FGF2 from dscECM-HP. As time progressed, the FGF2-dscECM-HP hydrogel exhibited a sustained-release of FGF2, with only $75 \%$ of the embedded FGF2 being released, even at 7 days. FGF2 embedded in the dscECM-HP hydrogel might go through two steps: first, release from the dscECM depot, and second, diffusion along the porous HP hydrogel to the external medium. The two processes dominated the release of FGF2 from dscECM-HP, resulting in its slow release profiles. Due to the strong affinity of FGF2 for the dscECM-HP hydrogel, $\sim 25 \%$ of the encapsulated FITC-FGF2 was retained in the hydrogel after 7 days of release. Our previous study ${ }^{18}$ indicated that the remaining FGF2 in dscECM-HP was still stable, and the remaining amount $(24.16 \% \pm 0.25 \%)$ determined by ELISA was identical to the inferred value.

\section{Cell proliferation and apoptosis in FGF2-dscECM-HP hydrogel in vitro}

As shown in Figure S2A, the survival rate of TG-injured PC12 cells was significantly improved after treatment with FGF2 solution or FGF2-HP hydrogel or FGF2-dscECM-HP. As expected, the FGF2-dscECM-HP hydrogel increased the survival rate of $\mathrm{PC} 12$ cells by $\sim 30 \%$. In contrast, neither the free dscECM nor the HP hydrogel matrix had any effect on the proliferation of TG-injured PC12 cells. These findings suggested that a suitable micro-niche provided by FGF2dscECM-HP might be important for cell survival and growth. As shown in Figure S3, the FGF2-dscECM-HP hydrogel promoted more cell attachment, migration, and infiltration into the hydrogel matrix compared to other groups. The migration and infiltration of the injured neuron toward damaged clefts where FGF2-dscECM-HP resided was beneficial for rapid joining of nerve fibers. Cell apoptosis was also assayed by flow cytometry. Similar results were exhibited in Figure S4. The apoptosis rate of PC12 cells was increased from $12.24 \%$ in the sham group to $31.39 \%$ after exposure of TG for $24 \mathrm{~h}$ (Figure S4). However, after treatment with the FGF2-dscECM-HP hydrogel, the apoptotic rate decreased to $16.21 \%$, suggesting the most effective inhibition of cell apoptosis.

\section{Morphological and functional recovery of injured spinal cord by FGF2-dscECM-HP hydrogel treatment}

FGF2 release from FGF2-dscECM-HP hydrogel in vivo To investigate the sustained-release of FGF2 at the damaged site, FITC-labeled FGF2 replaced native FGF2 and was embedded in the dscECM-HP hydrogel. The fluorescence imaging of the spinal cord after administration with the FITCFGF2-dscECM-HP hydrogel was detected using the Maestro in vivo optical imaging system at different time intervals. The results are shown in Figure 3. FGF2 was immediately released within 15 min after administration of the FGF-2-HP hydrogel, exhibiting strong fluorescence near the damaged site, whereas the fluorescence of the FGF2-dscECM-HP hydrogel only localized at the damaged site without diffusing into the surrounding zone. In addition, the fluorescence of FGF2-HP was uniformly distributed along the spinal cord
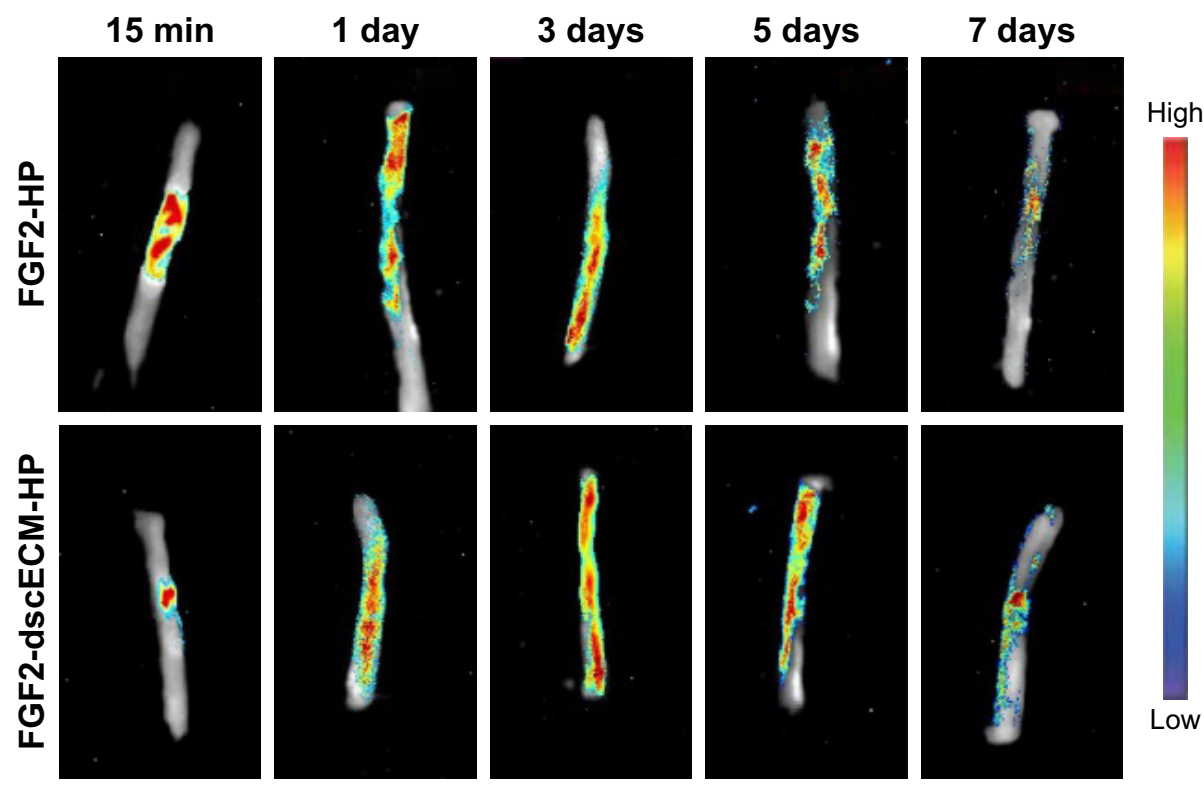

Figure 3 In vivo FGF2 retention in injured spinal cord after administration of FGF2-dscECM-HP hydrogel or FGF2-HP hydrogel. More red color indicates more FITC-FGF2 area. Abbreviations: FGF2, fibroblast growth factor-2; dscECM, decellular spinal cord extracellular matrix; HP, heparin-poloxamer; FITC, fluorescein isothiocyanate. 
over time, and FGF2 was completely released on the third day, exhibiting the strongest fluorescence at the bulk spinal cord. However, the surrounding fluorescence near the damaged site was observed on the first day after administration of the FGF2-dscECM-HP hydrogel, indicating no burst release of FGF2. The fluorescence of the FGF2-dscECM-HP hydrogel gradually decreased, and even on the seventh day, there was strong fluorescence at the damaged site, indicating the sustained-release of FGF2 at the administered site. These results were identical to those of the in vitro release study.

\section{Behavior movement}

The BBB rating scale after SCI is generally used to investigate functional recovery. ${ }^{33}$ The sham group exhibited normal BBB scores. The BBB scores showed that all rats (except for the sham group) suffered from partly paraplegic symptoms in the hind limbs (BBB score $=0$ at 1 day after SCI). The paralysis of the hind limbs of rats post-injury after 1 day is exhibited in Figure 4A. After a week of treatment, three joints of the hind limb could be active in the FGF2-dscECM-HP hydrogel group. The sole was located in the weight-bearing position or occasionally/frequently/continued to walk with the foot back, not walking with the weight-bearing foot after injury at 14 days in the FGF2-dscECM-HP hydrogel group.
At 1 and 3 days after treatment, there was no significant difference in BBB scores between the treatment groups and the SCI group. However, 14 and 28 days after treatment, the FGF2 treatment groups, both FGF-2 and FGF-2-dsc-HP, showed an increase in the BBB scores relative to the SCI group $(P<0.01$; Figure 4B) and dscECM-HP hydrogel groups, indicating the restoration of hind leg movement. Moreover, as expected, the FGF2-dscECM-HP hydrogel group showed a more significant effect than the FGF2 group. Similar results were observed for the angle of incline test. The FGF2-dscECM-HP group showed the most significant effect followed by the FGF2 group ( $P<0.01$, Figure 4C). At 28 days after treatment, the rat treated with FGF2dscECM-HP exhibited coordinated crawling and almost achieved the functional levels of the control group, whereas rats in the SCI group were still dragging their hind legs. These results suggested that the FGF2-dscECM-HP hydrogel could enhance the therapeutic effect of FGF2 on the functional improvement of locomotor activity.

\section{Morphological recovery and neuron regeneration of injured spinal cord}

Histopathological and pathological morphology of the injured spinal cord was evaluated by HE, Nissl, and
A
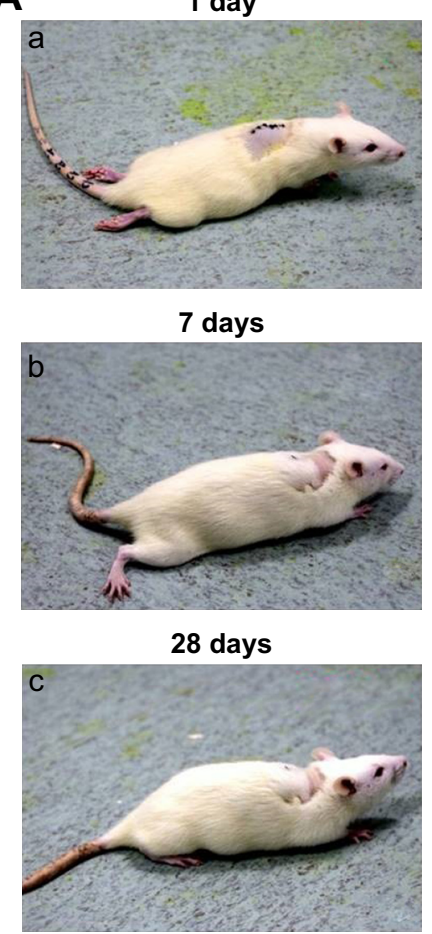
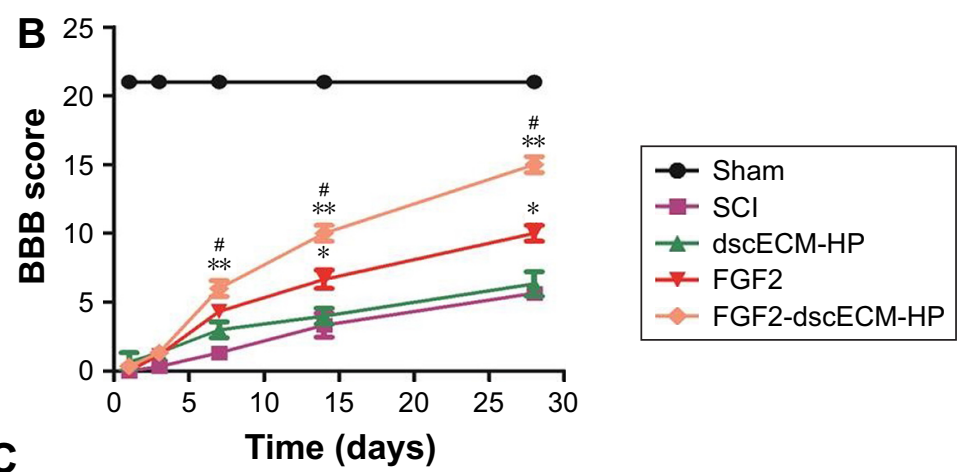

C

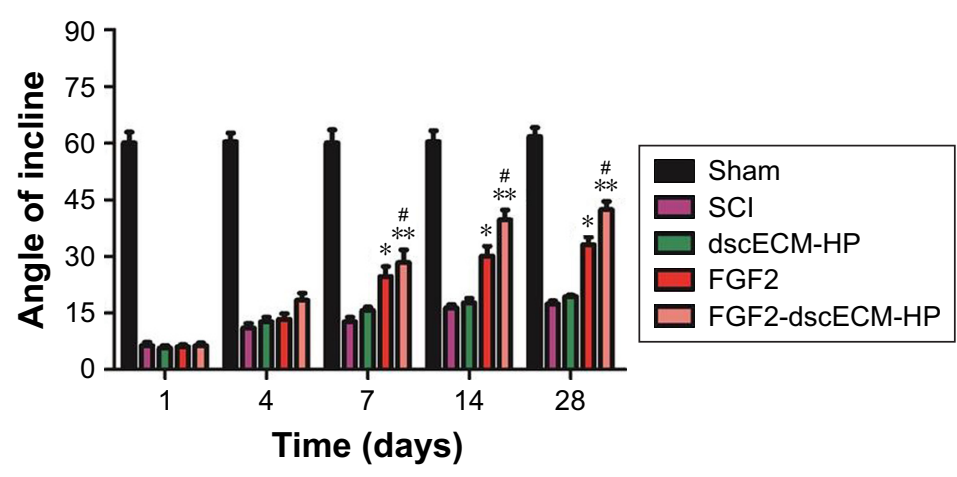

Figure 4 Motor function locomotion assessments of SCl rats: (A) behavior movement of hind limbs of SCl rats at (a) I, (b) 7, and (c) 28 days after treatment with FGF2dscECM-HP hydrogel, (B) BBB scores, and (C) angle of incline of SCl rats after various treatments at different times. Data were presented as mean \pm SD, $n=6$. FGF2-dscECM$\mathrm{HP}$ group versus $\mathrm{SCl}$ group $\left({ }^{*} P<0.05\right.$; $\left.* * P<0.01\right)$, FGF2-dscECM-HP group versus FGF2 group $\left({ }^{*} P<0.05\right)$.

Abbreviations: SCl, spinal cord injury; FGF2, fibroblast growth factor-2; dscECM, decellular spinal cord extracellular matrix; HP, heparin-poloxamer; BBB, Basso, Beattie, and Bresnahan. 
Immunofluorescence staining. Both the SCI group and dscECM-HP group exhibited an obvious cavity, as shown in Figure S5, and cell proliferation was lower during the recovery process. In contrast, the wound at the damaged site was healed significantly after treatment with free FGF2-HP or FGF2-dscECM-HP hydrogel, showing significant cell aggregation and proliferation. Specifically, in the FGF2dscECM-HP hydrogel group, the injured spinal cord fit together and exhibited a dense cell morphology, indicating a good bridge between the normal cells and the promotion of the mysterious link between cells.

The Nissl staining was used to evaluate the Nissl bodies of injured neurons in the rat spinal cord. As shown in Figure 5A, Nissl bodies were evidently reduced in SCI groups 14 days after injury compared to the sham group. By contrast, Nissl bodies were significantly increased after treatment with different FGF2 formulations, especially FGF2-dscECM-HP. Similarly, neuron-specific marker neuronal nuclei (NeuN) were also stained using immunofluorescence, and the results are shown in Figure 5B. NeuN-positive cells also decreased significantly 14 days after injury, but they were increased in rats treated with various FGF2-containing formulations.
Moreover, NeuN-positive cells in the FGF2-dscECM-HP hydrogel group were significantly greater than those in the FGF2 solution group, indicating its protective effect on neurons. However, neuron neurofilament (NF) proteins, including NF68, NF140, and NF200, indicated repair regeneration of injured neurons. ${ }^{34}$ NF200 was also detected using immunofluorescence, and the results are shown in Figure 5C. NF200-positive fibers in SCI group were obviously degraded, and the axons were broken. NF200 expression around the lesion in the FGF2 solution group was greater than that in the SCI group. Moreover, NF200 in the FGF2-dscECM-HP hydrogel group was much greater than that in the other injured groups, indicating obvious neuron regeneration around the lesion zone.

The axonal regeneration of the injured nerve was observed using the nerve tracing technique with BDA. ${ }^{35}$ Spinal cord stage sections below the injured site (T12-L1 plane) showed a significant decrease in the SCI group compared to the sham group (Figure 5D). All FGF2-containing groups showed enhanced green fluorescence compared to the SCI group. Moreover, the FGF2-dscECM-HP hydrogel group exhibited the highest integral optical density of positive BDA among
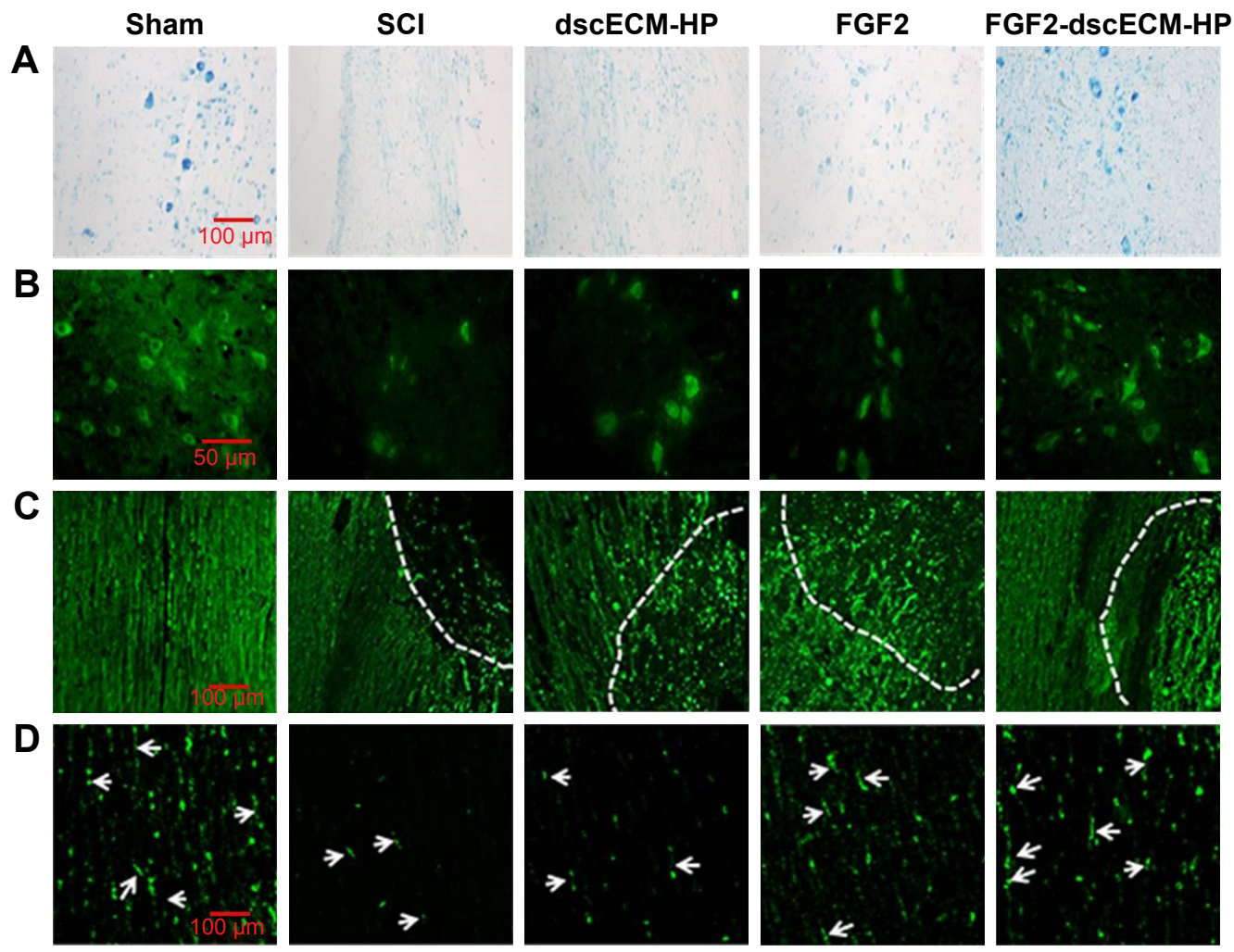

Figure 5 Repair effect of nerve fiber and axonal regeneration of $\mathrm{SCl}$ model rats 28 days after treatments: (A) Nissl staining of longitudinal slide position around damage site after treatments $(\times 200)$, (B) immunofluorescence staining of neuron $(\times 400)$, (C) immunofluorescence staining of neurofilament 200 (NF-200) in longitudinal slide position around the damage site $(\times 200$; dashed lines indicate the injured border), and (D) immunofluorescence staining of BDA protein after different treatments $(\times 200$; white arrows indicate the neonatal axon).

Abbreviations: SCl, spinal cord injury; BDA, biotinylated dextran amine; FGF2, fibroblast growth factor-2; dscECM, decellular spinal cord extracellular matrix; HP, heparinpoloxamer. 
A
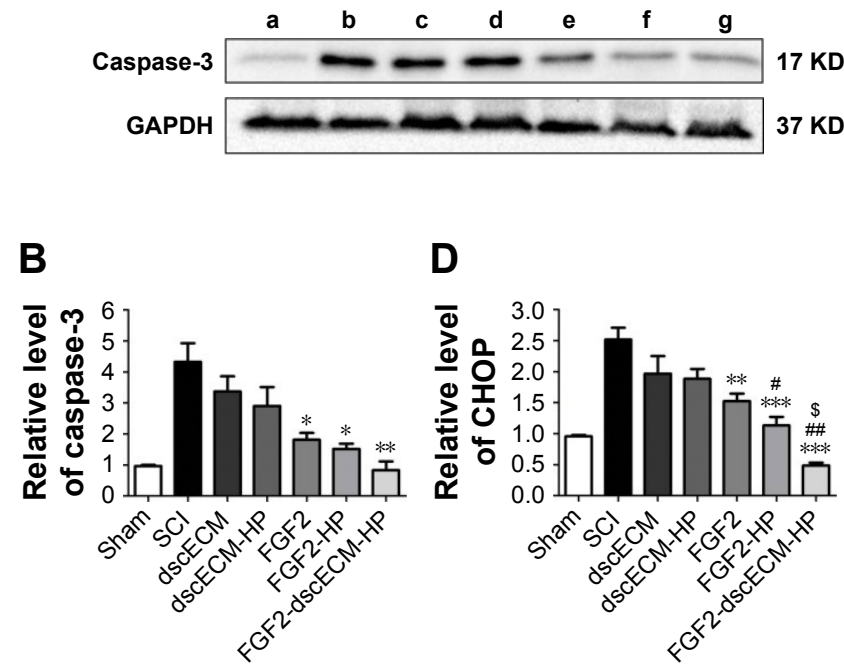

D

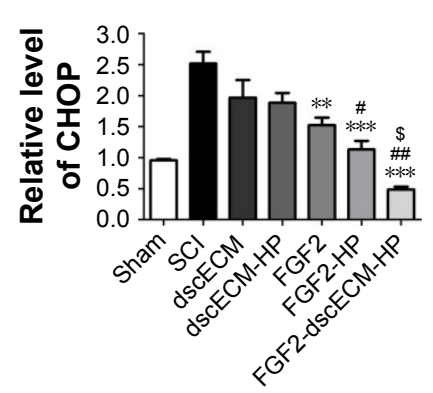

C

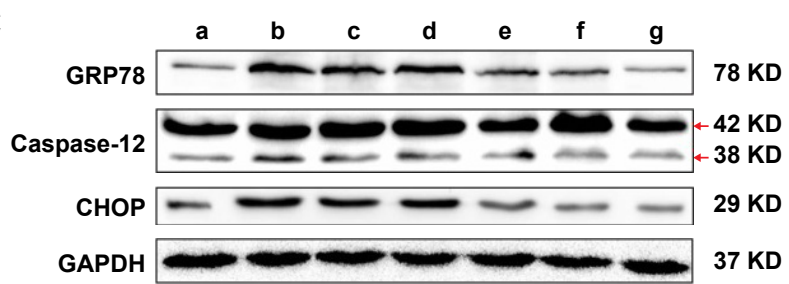

$\mathrm{E}$

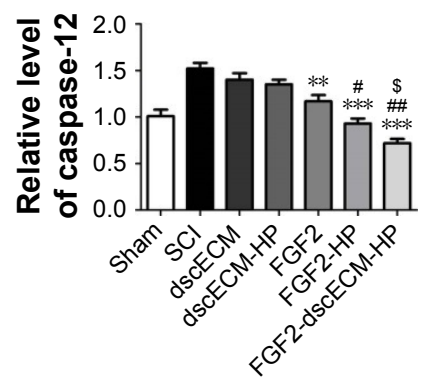

$\mathbf{F}$



Figure 6 In vivo expression of proteins associated with endoplasmic reticulum stress 28 days after treatments: (A) expression of caspase-3 detected by Western blot, (B) quantitative analysis of caspase-3 expression in Western blot, (C) expression level of GRP78, caspase-12, and CHOP detected by Western blot, and (D-F) quantitative analysis of CHOP, caspase-12, and GRP78 according to the results of Western blotting. a: Sham group; b: SCl group; c: dscECM group; d: dscECM + HP group; e: FGF2 solution group; f: FGF2-HP hydrogel group; and g: FGF2-dscECM-HP hydrogel group. Data were presented as mean $\pm \mathrm{SD}, \mathrm{n}=6$. FGF2-dscECM-HP group versus $\mathrm{SCl}$ group $(* P<0.05$; $* * P<0.01$;



Abbreviations: $\mathrm{SCl}$, spinal cord injury; FGF2, fibroblast growth factor-2; dscECM, decellular spinal cord extracellular matrix; HP, heparin-poloxamer.

these treatment groups, indicating its stronger therapeutic effect on the axonal regeneration.

In vivo cell apoptosis inhibited by FGF2-dscECM-HP To confirm inhibition of FGF2 on cell apoptosis after SCI, expression of caspase-3 was detected by immunohistochemical staining and Western blot. As shown in Figure 6A, caspase-3-positive cells in the SCI group increased significantly (Figure S6A) and caspase-3 was also highly expressed $14 \mathrm{~d}$ after injury. Caspase-3 was slightly decreased in the dscECM group and dscECM-HP hydrogel group but was not significantly different from the SCI group (Figure 6B) $(P>0.05)$. In contrast, treatments with FGF2 formulations could not only significantly decrease capase-3-positive cells but also inhibited the expression of capase- 3 reflected by the Western blot, indicating obvious inhibition of cell apoptosis. As expected, the FGF2-dscECM-HP hydrogel exhibited the most effective inhibition of cell apoptosis among various FGF2 formulations $(P<0.01)$.

\section{Inhibition of ER stress signal pathways after treatment with FGF2-dscECM-HP hydrogel}

CHOP protein was highly associated with ER stress-induced apoptosis, as reported in the literature. ${ }^{36} \mathrm{SCI}$ rats exhibited a significant number of CHOP-positive cells, indicating ER stress-induced apoptosis of cells after SCI (Figure S6B).
After treatment with FGF2 formulations, the number of CHOP-positive cells significantly decreased. Moreover, comparing FGF2 solutions, a lower expression of CHOP for FGF2-HP and FGF2-dscECM hydrogel groups was observed $(P<0.05$, Figure 6D). However, rats treated with FGF2dscECM-HP hydrogel exhibited the most effective inhibition of ER stress-induced apoptosis in vivo. In addition, the other two important proteins (GRP78 and Caspase-12) involved in endoplasmic reticulum stress were also detected using Western blot. The results were consistent with the level of $\mathrm{CHOP}$ and were even more pronounced (Figure 6C). These protein levels in rats treated with FGF2-dscECM-HP were relatively lower than those in the FGF2-HP group $(P<0.05$, Figure $6 \mathrm{E}$ and $\mathrm{F}$ ). These results suggested that the inhibitory role of FGF2-dscECM-HP hydrogel in endoplasmic reticulum stress was more obvious and long-lasting than in the FGF2-HP hydrogel.

\section{Discussion}

SCI registries from high-income countries and a meta-analysis of studies reporting the incidence of the disease allow us to estimate that worldwide, $\sim 250,000-500,000$ people sustain an SCI every year. ${ }^{37}$ Damage to the spinal cord characteristically causes inflammation, glial scarring, loss of nervous tissue and axons, and functional impairments. ${ }^{12,38}$ SCI remains a worldwide clinical challenge because there is still no viable therapeutic method. Neurotrophic factors, for example, nerve 
growth factor, neurotrophin 3, glial-derived neurotrophic factor, and FGF2, have been tested to promote anatomical and functional regeneration after SCI by local or systemic administration. ${ }^{39,40}$ However, the therapeutic effects for these neurotrophic factors are limited because of their low availability to injured spinal cords and their short half-life in vivo. In this study, we focus on developing a comprehensive means to repair the injured spinal cord and promote tissue regeneration. Three-dimensional hydrogel is an ideal carrier for protein-based therapeutics due to several advantages. For example, sustained delivery of therapeutic proteins by using hydrogel-forming biomaterials may support the cleft in injured tissue and maintain a beneficial microenvironment to facilitate cell attachment, growth, and migration, which is essential for rapid tissue restoration.

Poloxamers are non-ionic, poly (ethylene oxide)-poly (propylene oxide)-poly (ethylene oxide) copolymers (PEOPPO-PEO) that form micellar solutions at low concentrations and clear thermoreversible hydrogels at high concentrations. These copolymers have been widely used in various drug delivery systems due to their advantages, such as low toxicity and biocompatibility. Heparin can stabilize various growth factors or cytokines through specific receptor binding and thus control their release behavior. In our previous study, a new HP conjugate was synthesized, and its physicochemical properties were investigated. ${ }^{19}$ We prepared a novel HP hydrogel that had high affinity for growth factors such as NGF and aFGF and exhibited a controlled phase-transition temperature suitable for in situ administration. HP hydrogel lacked a micro-niche to store and stabilize growth factors as they were secreted in the body, making them incapable of attracting or attaching growing cells to injured sites in vivo, which is required to promote the regeneration of injured tissues. dscECM has been demonstrated to be a crucial element to promote adhesion, trophic support, and survival of growing cells. ${ }^{41}$ In addition, GAGs, an important component of dscECM, exhibited a high affinity for growth factors, which could serve as a micro-depot for storage and stabilization. ${ }^{42}$

In this study, a hybrid hydrogel was constructed by incorporating dscECM into the HP solution. After decellularization of normal spinal cord, dscECM maintained the essential components of normal spinal cord, in which the contents of collagen IV, laminin, and fibronectin were comparable to those of normal spinal cord reported in the literature. ${ }^{43}$ Moreover, dscECM retained a high level of GAGs, reaching $2.09 \pm 0.12 \mu \mathrm{g}$ GAGs/mg dry weight. The dscECM exhibited a strong affinity to FGF2, which resulted in a hybrid dscECM-HP hydrogel that adsorbed as much as double FGF-2 compared to HP hydrogel alone. Importantly, when FGF2 was preincubated with dscECM and incorporated into a cold HP solution, the ternary FGF2-dscECM-HP system displayed a special gel-solution-gel phase transition behavior similar to poloxamer 407. With a sponge-like structure, the FGF2-dscECM-HP hydrogel (16\%, w/v) had a suitable gelation temperature and apparent viscosity suitable for in situ application.

Upon in situ administration of FGF2-dscECM-HP to SCI model rats, morphological and functional recovery of injured spinal cord was observed at 28 days after treatment. Both the neuroprotective effect and neuron regeneration of FGF2dscECM-HP were enhanced by promoting cell proliferation and apoptotic inhibition. The survival rate of TG-injured PC12 cells was significantly improved after treatment with FGF2-dscECM-HP. As expected, the FGF2-dscECM-HP hydrogel increased the survival rate of $\mathrm{PC} 12$ cells by $\sim 30 \%$. The main purpose of the treatment of SCI was to delay the injury and death of injured neurons. Several reports have suggested that apoptosis plays a pivotal role in neuron death after SCI in animals or humans. ${ }^{44,45}$ First, inhibition of cell apoptosis has been detected in TG-induced injured PC12 cells. The results suggested that the neuroprotective effect of the FGF2-dscECM-HP hydrogel was related to the inhibition of ER stress-induced apoptosis.

Accordingly, a variety of mechanisms, such as disturbed ER stress, oxidative stress, inflammatory response, and ionic homeostasis, were involved in the progression of SCI-induced secondary SCI. ${ }^{46}$ Under stressful situations, the ER environment was compromised, and protein maturation was impaired. ER stress usually caused misfolded proteins to accumulate and triggered a characteristic stress response known as the unfolded protein response (UPR). UPR protects cells from stress and contributes to the re-establishment of cellular homeostasis. However, during prolonged ER stress, continuous UPR activation can promote cell death. ${ }^{47}$ After SCI, prolonged ER stress with the failure of cellular protective mechanisms by UPR eventually resulted in neural apoptosis. The expression level of ER stress markers CHOP, GRP78, and Caspase-12 was almost undetectable under physiological conditions. However, their expression levels were upregulated when protein folding at the ER was disrupted. ${ }^{48}$ In this study, the neuroprotective effect of FGF2-dscECM-HP was related to the inhibition of chronic ER stress-induced apoptosis. The survival of sensory neurons and axon regeneration in spinal cord lesions was also highly associated with the inhibition of endoplasmic reticulum stress. 


\section{Conclusion}

Compared to the FGF2 solution or FGF2-HP hydrogel, better therapeutic effect of FGF2-dscECM-HP was attributable to the following reasons. First, because of high affinity to FGF2, dscECM could serve as a micro-depot for storage and stabilization, which resulted in slower release of FGF2 from the FGF2-dscECM-HP hydrogel. Second, FGF2-dscECM-HP could fill the cleft of injured spinal cord and maintain a beneficial microenvironment to facilitate cell attachment, growth, and migration. The migration and infiltration of the injured neuron toward the damaged cleft where FGF2dscECM-HP resided was beneficial for rapid joining of nerve fibers. In summary, our research demonstrated that a hybrid FGF2-dscECM hydrogel may be a potential carrier to deliver macromolecular proteins to the injured site and enhance their therapeutic effects.

\section{Acknowledgments}

This research was supported by the National Natural Science Foundation of China (grant numbers 81571392, 81603036, 81360195 and 81772316), Key Research and Development Program of Zhejiang Province (grant number 2018C03013), Zhejiang Provincial Program for the Cultivation of Highlevel Innovative Health Talents (Ying-Zheng Zhao), 151 Talent Project of Zhejiang Province and 551 Talent Project of Wenzhou (Ying-Zheng Zhao), Key Support of High-level Talent Innovation and Technology project of Wenzhou (Ying-Zheng Zhao), and School Talent Start Fund of Wenzhou Medical University (grant number QTJ15020).

\section{Disclosure}

The authors report no conflicts of interest in this work.

\section{References}

1. Varma AK, Das A, Wallace GT, et al. Spinal cord injury: a review of current therapy, future treatments, and basic science frontiers. Neurochem Res. 2013;38(5):895-905

2. Penas C, Guzman MS, Verdu E, Fores J, Navarro X, Casas C. Spinal cord injury induces endoplasmic reticulum stress with different cell-type dependent response. J Neurochem. 2007;102(4):1242-1255.

3. Yong CS, Choi JS, Quan QZ, et al. Effect of sodium chloride on the gelation temperature, gel strength and bioadhesive force of poloxamer gels containing diclofenac sodium. Int J pharmaceut. 2001;226(1-2): 195-205.

4. Moon YJ, Lee JY, Oh MS, et al. Inhibition of inflammation and oxidative stress by Angelica dahuricae radix extract decreases apoptotic cell death and improves functional recovery after spinal cord injury. $J$ Neurosci Res. 2012;90(1):243-256.

5. Goldshmit Y, Frisca F, Pinto AR, et al. Fgf2 improves functional recovery-decreasing gliosis and increasing radial glia and neural progenitor cells after spinal cord injury. Brain Behav. 2014;4(2):187-200.

6. Xie JX, Feng Y, Yuan JM, et al. Positive effects of bFGF modified rat amniotic epithelial cells transplantation on transected rat optic nerve. PLoS One. 2015;10(3):e0119119.
7. Karimi-Abdolrezaee S, Schut D, Wang J, Fehlings MG. Chondroitinase and growth factors enhance activation and oligodendrocyte differentiation of endogenous neural precursor cells after spinal cord injury. PLoS One. 2012;7(5):e37589.

8. Beenken A, Mohammadi M. The FGF family: biology, pathophysiology and therapy. Nat Rev Drug Discov. 2009;8(3):235-253.

9. Ye LB, Yang Y, Zhang X, et al. The role of bFGF in the excessive activation of astrocytes is related to the inhibition of TLR4/NF kappa B signals. Int J Mol Sci. 2016;17(1):37.

10. Mantilla CB, Gransee HM, Zhan WZ, Sieck GC. Motoneuron BDNF/ TrkB signaling enhances functional recovery after cervical spinal cord injury. Exp Neurol. 2013;247:101-109.

11. Parr AM, Tator $\mathrm{CH}$. Intrathecal epidermal growth factor and fibroblast growth factor-2 exacerbate meningeal proliferative lesions associated with intrathecal catheters. Neurosurgery. 2007;60(5):926-933; discussion 926-933.

12. Gumera C, Rauck B, Wang YD. Materials for central nervous system regeneration: bioactive cues. J Mater Chem. 2011;21(20):7033-7051.

13. Sayer FT, Oudega M, Hagg T. Neurotrophins reduce degeneration of injured ascending sensory and corticospinal motor axons in adult rat spinal cord. Exp Neurol. 2002;175(1):282-296.

14. Bregman BS, McAtee M, Dai HN, Kuhn PL. Neurotrophic factors increase axonal growth after spinal cord injury and transplantation in the adult rat. Exp Neurol. 1997;148(2):475-494.

15. Tian JL, Zhao YZ, Jin Z, et al. Synthesis and characterization of Poloxamer 188-grafted heparin copolymer. Drug Dev Ind Pharm. 2010; 36(7):832-838.

16. Zhao YZ, Jiang X, Xiao J, et al. Using NGF heparin-poloxamer thermosensitive hydrogels to enhance the nerve regeneration for spinal cord injury. Acta Biomater. 2016;29:71-80.

17. Medberry CJ, Crapo PM, Siu BF, et al. Hydrogels derived from central nervous system extracellular matrix. Biomaterials. 2013;34(4): 1033-1040.

18. Xu HL, Tian FR, Lu CT, et al. Thermo-sensitive hydrogels combined with decellularised matrix deliver bFGF for the functional recovery of rats after a spinal cord injury. Sci Rep. 2016;6:38332.

19. Zhao YZ, Lv HF, Lu CT, et al. Evaluation of a novel thermosensitive heparin-poloxamer hydrogel for improving vascular anastomosis quality and safety in a rabbit model. PLoS One. 2013;8(8):e73178.

20. Zhao YZ, Jiang X, Xiao J, et al. Using NGF heparin-poloxamer thermosensitive hydrogels to enhance the nerve regeneration for spinal cord injury. Acta Biomater. 2016;29:71-80.

21. Niu GG, Zhang HB, Song L, et al. Thiol/acrylate-modified PEOPPO-PEO triblocks used as reactive and thermosensitive copolymers. Biomacromolecules. 2008;9(10):2621-2628.

22. Zhao YZ, Tian XQ, Zhang M, et al. Functional and pathological improvements of the hearts in diabetes model by the combined therapy of bFGF-loaded nanoparticles with ultrasound-targeted microbubble destruction. J Control Release. 2014;186:22-31.

23. Robert MC, Frenette M, Zhou C, et al. A drug delivery system for administration of anti-TNF-alpha antibody. Transl Vis Sci Technol. 2016;5(2):11.

24. Ansorena E, De Berdt P, Ucakar B, et al. Injectable alginate hydrogel loaded with GDNF promotes functional recovery in a hemisection model of spinal cord injury. Int J Pharm. 2013;455(1-2):148-158.

25. Zhang M, Yu WZ, Shen XT, et al. Advanced interfere treatment of diabetic cardiomyopathy rats by aFGF-loaded heparin-modified microbubbles and UTMD technique. Cardiovasc Drugs Ther. 2016;30(3):247-261.

26. Lan L, Tian FR, ZhuGe DL, et al. Implantable porous gelatin microspheres sustained-release of bFGF and improved its neuroprotective effect on rats after spinal cord injury. PLoS One. 2017;12(3):e0173814.

27. Dinh P, Hazel A, Palispis W, Suryadevara S, Gupta R. Functional assessment after sciatic nerve injury in a rat model. Microsurgery. 2009; 29(8):644-649.

28. Weidner N, Grill RJ, Tuszynski MH. Elimination of basal lamina and the collagen "scar" after spinal cord injury fails to augment corticospinal tract regeneration. Exp Neurol. 1999;160(1):40-50. 
29. Coolen LM, Jansen HT, Goodman RL, Wood RI, Lehman MN. A new method for simultaneous demonstration of anterograde and retrograde connections in the brain: co-injections of biotinylated dextran amine and the beta subunit of cholera toxin. J Neurosci Methods. 1999; 91(1-2):1-8.

30. Fiebich BL, Schleicher S, Butcher RD, Craig A, Lieb K. The neuropeptide substance $\mathrm{P}$ activates $\mathrm{p} 38$ mitogen-activated protein kinase resulting in IL-6 expression independently from NF-kappa B. J Immunol. 2000;165(10):5606-5611.

31. Viswanath A, Vanacker J, Germain L, et al. Extracellular matrixderived hydrogels for dental stem cell delivery. J Biomed Mater Res A. 2017;105(1):319-328.

32. Jiang T, Ren XJ, Tang JL, Yin H, Wang KJ, Zhou CL. Preparation and characterization of genipin-crosslinked rat acellular spinal cord scaffolds. Mater Sci Eng C Mater Biol Appl. 2013;33(6):3514-3521.

33. Basso DM, Beattie MS, Bresnahan JC. A sensitive and reliable locomotor rating scale for open field testing in rats. J Neurotrauma. 1995; 12(1):1-21.

34. Liu WG, Wang ZY, Huang ZS. Bone marrow-derived mesenchymal stem cells expressing the bFGF transgene promote axon regeneration and functional recovery after spinal cord injury in rats. Neurol Res. 2011; 33(7):686-693.

35. Chen P, Goldberg DE, Kolb B, Lanser M, Benowitz LI. Inosine induces axonal rewiring and improves behavioral outcome after stroke. Proc Natl Acad Sci U S A. 2002;99(13):9031-9036.

36. Wang Z, Zhang C, Hong Z, Chen H, Chen W, Chen G. C/EBP homologous protein (CHOP) mediates neuronal apoptosis in rats with spinal cord injury. Exp Ther Med. 2013;5(1):107-111.

37. Furlan JC. Databases and registries on traumatic spinal cord injury in Canada. Can J Neurol Sci. 2013;40(4):454-455.

38. Wang Q, He Y, Zhao Y, et al. A thermosensitive heparin-poloxamer hydrogel bridges aFGF to treat spinal cord injury. ACS Appl Mater Interfaces. 2017;9(8):6725-6745.
39. Romero MI, Rangappa N, Garry MG, Smith GM. Functional regeneration of chronically injured sensory afferents into adult spinal cord after neurotrophin gene therapy. J Neurosci. 2001;21(21):8408-8416.

40. Zhang Y, Dijkhuizen PA, Anderson PN, Lieberman AR, Verhaagen J. NT-3 delivered by an adenoviral vector induces injured dorsal root axons to regenerate into the spinal cord of adult rats. J Neurosci Res. 1998; 54(4):554-562.

41. Sun CC, Chou SF, Lai JY, Cho CH, Lee CH. Dependence of corneal keratocyte adhesion, spreading, and integrin beta1 expression on deacetylated chitosan coating. Mater Sci Eng C. 2016;63:222-230.

42. Gasiorowski JZ, Murphy CJ, Nealey PF. Biophysical cues and cell behavior: the big impact of little things. Annu Rev Biomed Eng. 2013; 15:155-176.

43. Crapo PM, Medberry CJ, Reing JE, et al. Biologic scaffolds composed of central nervous system extracellular matrix. Biomaterials. 2012; 33(13):3539-3547.

44. Shuman SL, Bresnahan JC, Beattie MS. Apoptosis of microglia and oligodendrocytes after spinal cord contusion in rats. J Neurosci Res. 1997;50(5):798-808.

45. Emery E, Aldana P, Bunge MB, et al. Apoptosis after traumatic human spinal cord injury. J Neurosurg. 1998;89(6):911-920.

46. Lupachyk S, Watcho P, Stavniichuk R, Shevalye H, Obrosova IG. Endoplasmic reticulum stress plays a key role in the pathogenesis of diabetic peripheral neuropathy. Diabetes. 2013;62(3):944-952.

47. Fernandez A, Ordonez R, Reiter RJ, Gonzalez-Gallego J, Mauriz JL. Melatonin and endoplasmic reticulum stress: relation to autophagy and apoptosis. J Pineal Res. 2015;59(3):292-307.

48. Schneider K, Bertolotti A. Surviving protein quality control catastrophes from cells to organisms. J Cell Sci. 2015;128(21):3861-3869.
International Journal of Nanomedicine

\section{Publish your work in this journal}

The International Journal of Nanomedicine is an international, peerreviewed journal focusing on the application of nanotechnology in diagnostics, therapeutics, and drug delivery systems throughout the biomedical field. This journal is indexed on PubMed Central, MedLine, CAS, SciSearch ${ }^{\circledR}$, Current Contents ${ }^{\circledR} /$ Clinical Medicine,

\section{Dovepress}

Journal Citation Reports/Science Edition, EMBase, Scopus and the Elsevier Bibliographic databases. The manuscript management system is completely online and includes a very quick and fair peer-review system, which is all easy to use. Visit http://www.dovepress.com/ testimonials.php to read real quotes from published authors. 